\title{
Preventing clinically important deterioration with single-inhaler triple therapy in COPD
}

Ian Naya ${ }^{1}$, Chris Compton ${ }^{1}$, Afisi S. Ismaila ${ }^{2,3}$, Ruby Birk $^{4}$, Noushin Brealey ${ }^{4}$, Maggie Tabberer ${ }^{4}$, Chang-Qing Zhu ${ }^{4}$, David A. Lipson ${ }^{5,6}$ and Gerard Criner $^{7}$

Affiliations: ${ }^{1}$ GSK, Brentford, UK. ${ }^{2}$ GSK, Research Triangle Park, NC, USA. ${ }^{3}$ Dept of Health Research Methods, Evidence and Impact, McMaster University, Hamilton, ON, Canada. ${ }^{4}$ GSK, Uxbridge, UK. ${ }^{5} \mathrm{GSK}$, King of Prussia, PA, USA. ${ }^{6}$ Perelman School of Medicine, University of Pennsylvania, Philadelphia, PA, USA. ${ }^{7}$ Lewis Katz School of Medicine, Temple University, Philadelphia, PA, USA.

Correspondence: Ian Naya, GSK Respiratory Medical Franchise, 980 Great West Road, Brentford, Middlesex, TW8 9GS, UK. E-mail: ian.p.nayalagsk.com

ABSTRACT Clinically important deterioration (CID) is a novel composite end-point (lung function, health status, exacerbations) for assessing disease stability in patients with chronic obstructive pulmonary disease (COPD)

We prospectively analysed CID in the FULFIL study. FULFIL (ClinicalTrials.gov NCT02345161; randomised, double-blind, double-dummy, multicentre study) compared 24 weeks of once daily, singleinhaler fluticasone furoate/umeclidinium/vilanterol (FF/UMEC/VI) 100/62.5/25 $\mu \mathrm{g}$ with twice daily budesonide/formoterol (BUD/FOR) $400 / 12 \mu \mathrm{g}$ in patients aged $\geqslant 40$ years with symptomatic advanced COPD (Global Initiative for Chronic Obstructive Lung Disease group D). A subset of patients received study treatment for up to 52 weeks. Time to first CID event was assessed over 24 and 52 weeks using two approaches for the health status component: St George's Respiratory Questionnaire and COPD assessment test. FF/UMEC/VI significantly reduced the risk of a first CID event by $47-52 \%$ versus BUD/FOR in the 24- and 52-week populations using both CID definitions $(\mathrm{p}<0.001)$.

The median time to first CID event was $\geqslant 169$ days and $\leqslant 31$ days with FF/UMEC/VI and BUD/FOR, respectively. Only stable patients with no CID at 24 weeks demonstrated sustained clinically important improvements in lung function and health status at 52 weeks versus those who had experienced CID.

Once daily, single-inhaler FF/UMEC/VI significantly reduced the risk of CID versus twice daily BUD/ FOR with a five-fold longer period without deterioration.

@ERSpublications

Fluticasone furoate/umeclidinium/vilanterol improves disease stability in COPD compared with budesonide/formoterol http://ow.ly/TaNY30loBa8

Cite this article as: Naya I, Compton C, Ismaila AS, et al. Preventing clinically important deterioration with single-inhaler triple therapy in COPD. ERJ Open Res 2018; 4: 00047-2018 [https:// doi.org/10.1183/23120541.00047-2018]. 


\section{Introduction}

Management guidelines for chronic obstructive pulmonary disease (COPD) advocate monitoring patients for any worsening in lung function, symptoms or exacerbations to determine the adequacy of treatment [1]. In COPD clinical trials, it is standard practise to evaluate the impact of therapies on improving lung function and patient-reported outcomes. However, there is often little focus on assessing disease stability, with the possible exception of monitoring for exacerbations. As many patients with COPD can show signs of acute or sustained disease deterioration over a relatively short time-course, maintaining stability for longer is likely to become increasingly important in patients with more advanced disease.

The Global Initiative for Chronic Obstructive Lung Disease (GOLD) guidelines recommend a long-acting muscarinic antagonist (LAMA) plus an inhaled corticosteroid (ICS)/long-acting $\beta_{2}$-agonist (LABA) for patients with advanced COPD with persistent symptoms and future exacerbation risk [1]. Treatment with a LAMA plus an ICS/LABA has been shown to improve lung function and health status, and reduce exacerbations in patients with COPD, compared with LAMA monotherapy or ICS/LABA dual therapy [2-6]. However, there have been relatively few longer term studies that have evaluated the benefit of triple therapy for COPD [4].

The pivotal phase III Lung FUnction and quality of LiFe assessment in COPD with closed trIpLe therapy (FULFIL) study demonstrated statistically significant and clinically meaningful improvements in trough forced expiratory volume in $1 \mathrm{~s}$ (FEV1) and St George's Respiratory Questionnaire (SGRQ) total score (co-primary end-points), as well as a statistically significant reduction in moderate/severe exacerbation rate, with once daily, single-inhaler fluticasone furoate/umeclidinium/vilanterol (FF/UMEC/VI), compared with twice daily budesonide/formoterol (BUD/FOR), in patients with symptomatic COPD at risk of exacerbations or with severe airflow limitation [7].

Recently, a novel composite end-point was introduced that aims to evaluate disease stability in patients with COPD, and includes components that measure time to a first moderate/severe exacerbation and/or clinically meaningful deterioration in lung function and/or decline in health status [8]. This composite end-point, referred to as clinically important deterioration (CID), has been assessed retrospectively in several clinical studies to establish the importance of aiming for maximum bronchodilation in patients with less advanced COPD [8-10]. However, to our knowledge, no COPD study has used this composite CID end-point as an a priori efficacy measure.

Here, we report the first results of a prospective analysis of CID in the FULFIL study. We aimed to assess CID events in patients with advanced COPD over 24 and 52 weeks of follow-up, and the impact of CID status on future outcomes, by analysing CID at 24 weeks in a subset of the intent-to-treat (ITT) population who received study treatment for up to 52 weeks. In addition, we evaluated CIDs using two different methods of assessing the health status component of the composite end-point: SGRQ total score (as has been used previously [8-10]) and COPD assessment test (CAT) score [11, 12].

\section{Methods}

\section{Study design}

FULFIL was a randomised, double-blind, double-dummy, parallel group, multicentre study (ClinicalTrials. gov NCT02345161; GSK study CTT116853) [7]. Patients were randomised to receive 24 weeks (ITT population) of once daily FF/UMEC/VI $100 \mu \mathrm{g} / 62.5 \mu \mathrm{g} / 25 \mu \mathrm{g}$ via the ELLIPTA inhaler (GSK) and twice daily placebo via the Turbuhaler (AstraZeneca), or twice daily BUD/FOR $400 \mu \mathrm{g} / 12 \mu \mathrm{g}$ via the Turbuhaler and once daily placebo via the ELLIPTA inhaler. The first 430 patients who were enrolled and consented to longer term treatment received blinded study treatment for up to 52 weeks (extension (EXT) population).

Patients aged $\geqslant 40$ years with COPD defined as GOLD group D (according to the 2015 GOLD report; FEV 1 $<50 \%$ and CAT $\geqslant 10$ or patients with $\mathrm{FEV}_{1} \geqslant 50 \%$ to $<80 \%$ and CAT $\geqslant 10$, and either at least two moderate exacerbations or at least one severe exacerbation in the past year) were eligible for enrolment. Patients were required to be receiving daily maintenance treatment for COPD for $\geqslant 3$ months prior to screening and to continue these medications unchanged during the 2-week run-in period. Key exclusion criteria included a current diagnosis of asthma causing their symptoms, unresolved pneumonia or a severe COPD exacerbation.

The FULFIL protocol was approved by relevant institutional review boards/ethics committees, and the study was conducted in accordance with International Conference on Harmonisation Good Clinical Practise guidelines and the Declaration of Helsinki. All patients provided written informed consent prior to enrolment.

\section{Study assessments}

The time to a first composite and individual CID event type was prospectively defined in the FULFIL trial. The time to a first composite CID event was assessed up to 24 weeks (ITT population) and up to 52 weeks 
(EXT population). Two definitions of the composite CID were used: 1) moderate/severe exacerbation and/ or $\geqslant 4$-unit deterioration from baseline in SGRQ total score and/or $\geqslant 100 \mathrm{~mL}$ deterioration from baseline in FEV1 (SGRQ-containing CID); and 2) moderate/severe exacerbation and/or $\geqslant 2$-unit deterioration from baseline in CAT score and/or $\geqslant 100 \mathrm{~mL}$ deterioration from baseline in FEV1 (CAT-containing CID). The occurrence of a CID was also assessed by individual CID component event type and patient subgroups: post hoc sensitivity analysis; GOLD grade (1/2 versus 3/4); exacerbation history in previous year $(0-1$ versus $\geqslant 2$, including one hospitalisation); sex; baseline CAT score $(<20$ versus $\geqslant 20)$; prior treatment with triple therapy (yes versus no) or ICS (yes versus no); and smoking status (current smoker versus former smoker).

The impact of CID status at 24 weeks was examined for the combined treatment groups using descriptive statistics on future exacerbation incidence, trough FEV1, SGRQ total score, CAT and Evaluating Respiratory Symptoms in COPD (E-RS:COPD) for the EXT population up to 52 weeks (up to 6 months of post-CID event follow-up). Two subgroups of patients were created based on CID status at 24 weeks: the CID-negative (CID-) subgroup included patients who had not experienced any CID by 24 weeks; and the CID-positive (CID+) subgroup included patients who had experienced at least one CID event by 24 weeks.

Potential COPD exacerbations were identified based on patient-reported symptoms in an eDiary and confirmed by follow-up with the investigator. Spirometry was performed at screening and at each clinic visit during the treatment period (day 1 (randomisation) and weeks 2, 4, 12 and 24 (and weeks 36 and 52 in the EXT population)) using standardised equipment according to American Thoracic Society/European Respiratory Society guidelines [13]. The SGRQ and CAT were completed by patients at day 1 and at weeks 4 and 24 (and week 52 in the EXT population) using an eDiary. The E-RS:COPD was completed each evening using the eDiary (in this article, E-RS:COPD data are only presented for the EXT population during weeks 25 to 52 for the analyses of the impact of 24-week CID status in follow-up).

\section{Statistical analyses}

The ITT population comprised all randomised patients, except those randomised in error. The EXT population comprised the subset of patients in the ITT population who were enrolled into the 52-week extension phase. The risk reduction for a first composite CID event was based on time-to-first-event analysis using a Cox proportional hazards model. Statistical analyses were performed using SAS (version 9.3; SAS Institute Inc, Cary, NC, USA). All data from all visits were used in the CID derivations. A sensitivity analysis, which excluded spirometry data collected at visits without corresponding information on health status, was also performed to examine the composite CID outcomes weighted equally for trough FEV1 and health status.

\section{Results}

\section{Patients}

A total of 1810 patients were included in the ITT population (FF/UMEC/VI: $n=911$; BUD/FOR: $n=899$ ) and 430 in the EXT population (FF/UMEC/VI: n=210; BUD/FOR: $n=220$ ). Patient demographics and baseline characteristics are shown in table 1 .

Time to a first composite CID event

In both the ITT and EXT populations, a clear separation between the treatment groups was apparent within 1 month, with a median $\geqslant 5$-fold greater period free from deterioration with FF/UMEC/VI compared with BUD/FOR (figure 1).

In the ITT population, the median time to a first composite CID event using the SGRQ-containing CID definition was 170 days with FF/UMEC/VI and 30 days with BUD/FOR. Similar results were shown using the CAT-containing CID definition: 169 and 30 days with FF/UMEC/VI and BUD/FOR, respectively. Treatment with FF/UMEC/VI significantly reduced the risk of a first composite CID over 24 weeks by $52 \%$ and $51 \%$ versus BUD/FOR using the SGRQ- and CAT-containing definitions, respectively $(\mathrm{p}<0.001)$ (figure 1a).

In the EXT population, the median time to a first composite CID event using the SGRQ-containing CID definition was 170 days with FF/UMEC/VI and 31 days with BUD/FOR. Using the CAT-containing CID definition, the median time to a first composite CID event was 255 and 31 days with FF/UMEC/VI and $\mathrm{BUD} / \mathrm{FOR}$, respectively. FF/UMEC/VI significantly reduced the risk of a first composite CID over 52 weeks by $48 \%$ and $47 \%$ versus BUD/FOR using the SGRQ- and CAT-containing definitions, respectively $(\mathrm{p}<0.001)$ (figure $1 \mathrm{~b})$.

The results of a sensitivity analysis, which censored deteriorations in FEV1 that were identified at visits without corresponding information on health status, were in full agreement with the ITT analyses (table 2). 


\section{TABLE 1 Patient characteristics at baseline}

\section{Characteristic}

ITT population 24 weeks

FF/UMEC/VI BUD/FOR
$100 / 62.5 / 25 \mu \mathrm{g}$

\section{0/12 $\mu \mathrm{g}$}

899
$63.7 \pm 8.7$

$236(26)$

$394(44)$

$39.2 \pm 22.2$

602 (67)

317 (35)

$253(28)$

329 (37)

99 (11)

$1339 \pm 480$

$45.1 \pm 13.6$

$50.8 \pm 16.7$

$12.97 \pm 5.93$
EXT population 52 weeks

\section{FF/UMEC/VI}

$100 / 62.5 / 25 \mu \mathrm{g}$

BUD/FOR

400/12 $\mu \mathrm{g}$

$\begin{array}{cc}210 & 220 \\ 63.7 \pm 7.8 & 63.3 \pm 8.4 \\ 53(25) & 58(26) \\ 95(45) & 97(44) \\ 39.8 \pm 19.9 & 39.6 \pm 23.1 \\ 144(69) & 152(69) \\ & \\ 62(30) & 72(33) \\ 77(37) & 79(36) \\ 71(34) & 69(31) \\ 18(9) & 20(9) \\ 1425 \pm 500 & 1368 \pm 510 \\ 47.1 \pm 13.3 & 45.4 \pm 14.9 \\ 53.0 \pm 16.1 & 50.8 \pm 15.5 \\ 13.54 \pm 5.44 & 13.00 \pm 5.58\end{array}$

Data are presented as mean \pm SD or $n(\%)$, unless otherwise stated. ITT: intent-to-treat; EXT: extension; FF: fluticasone furoate; UMEC: umeclidinium; VI: vilanterol; BUD: budesonide; FOR: formoterol; COPD: chronic obstructive pulmonary disease; FEV1: forced expiratory volume in $1 \mathrm{~s}$; SGRQ: St George's Respiratory Questionnaire; E-RS:COPD: Evaluating Respiratory Symptoms in COPD. \#: cardiovascular risk factors included, but were not limited to, hypertension, hypercholesterolemia, coronary heart disease and diabetes mellitus.

Occurrence of CID events by individual CID component event type

In the ITT population, treatment with FF/UMEC/VI significantly reduced the risk of a CID in lung function by $65 \%$ (95\% CI $60-70 ; \mathrm{p}<0.001)$, a first moderate/severe exacerbation by $33 \%$ (95\% CI $12-48 ; \mathrm{p}=0.004)$ and a deterioration in health status measured by SGRQ (25\% risk reduction (95\% CI $12-36 ; \mathrm{p}<0.001))$ or by CAT (22\% risk reduction (95\% CI 8-33; $\mathrm{p}=0.003)$ ), compared with BUD/FOR (figure $2 \mathrm{a}$ and $\mathrm{b}$ ).

In the smaller EXT population, FF/UMEC/VI significantly reduced the risk of a CID event in lung function by $62 \%$ (95\% CI 49-72; $\mathrm{p}<0.001)$ and exacerbations by $46 \%$ (95\% CI 17-64; $\mathrm{p}=0.005)$, compared with BUD/FOR. For the health status component, there was a numerical reduction in the risk of a CID with FF/UMEC/VI versus BUD/FOR using both the SGRQ (23\% risk reduction (95\% CI $-2-43 ; \mathrm{p}=0.072)$ ) and CAT (19\% risk reduction (95\% CI $-8-40 ; \mathrm{p}=0.143)$ ) (figure $2 \mathrm{c}$ and $\mathrm{d}$ ).

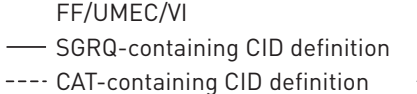

a)

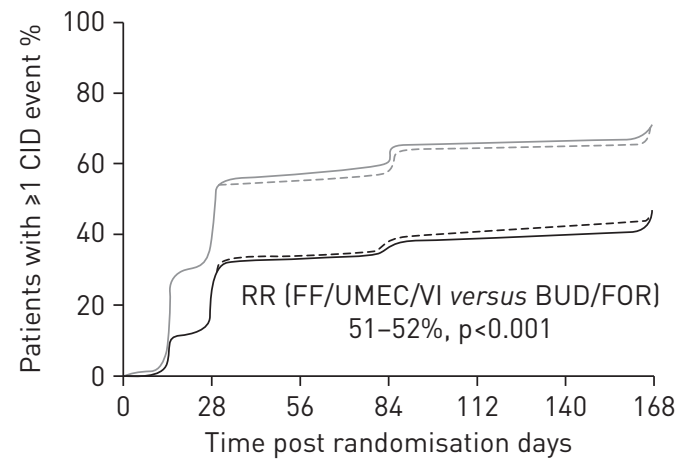

BUD/FOR

SGRQ-containing CID definition

- CAT-containing CID definition

b)

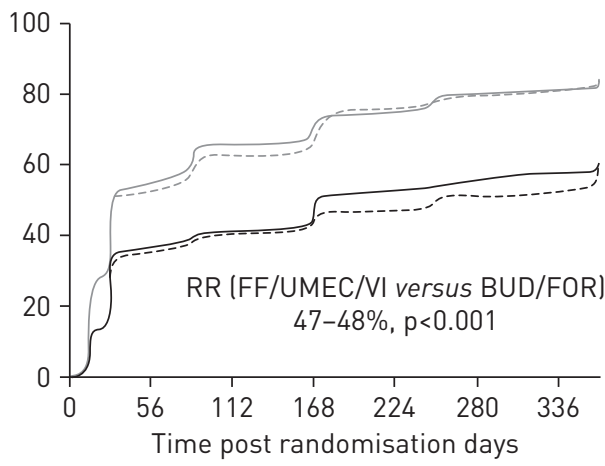

FIGURE 1 Time to a first composite clinically important deterioration (CID) event in a) the intent-to-treat population (24-week study duration, $n=1810$ ) and b) the extension population (52-week study duration, $n=430$ ). RR: risk reduction (based on a time-to-first-event analysis using a Cox proportional hazards model); FF: fluticasone furoate; UMEC: umeclidinium; VI: vilanterol; BUD: budesonide; FOR: formoterol; SGRQ: St George's Respiratory Questionnaire; CAT: chronic obstructive pulmonary disease assessment test. 
TABLE 2 Results of the sensitivity analysis, excluding spirometry data, collected at visits without corresponding health status

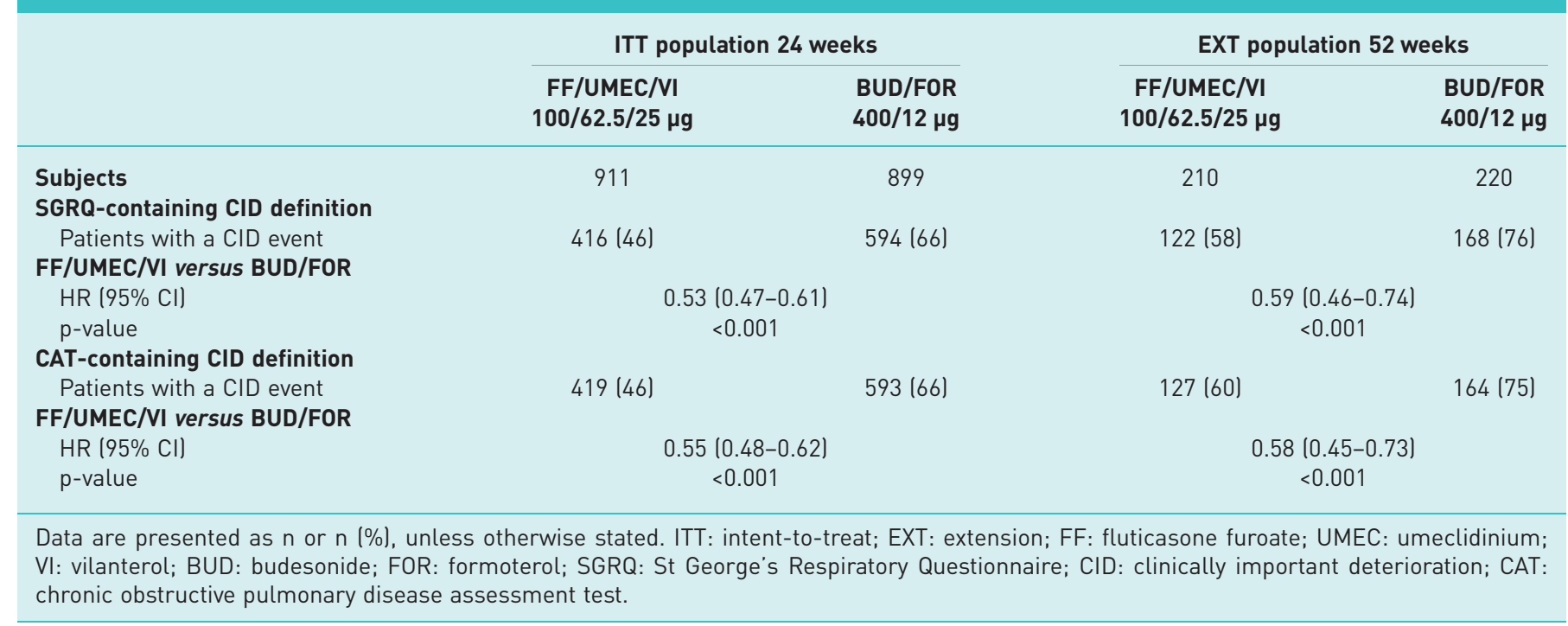

Time to a first composite CID event in different patient subgroups

Sensitivity analyses of the time to a first composite CID event in ITT patient subgroups for both CID definitions, segmented by baseline severity of airway obstruction, health status impact, exacerbation history, sex and prior ICS and triple therapy at screening, showed concordant results to the overall ITT analysis (figure $3 \mathrm{a}$ and $\mathrm{b}$ ). However, we identified a significant interaction by smoking status (pinteraction $=0.006$ and pinteraction $=0.030$, using the SGRQ- and CAT-containing CID definitions, respectively), indicating that the efficacy benefit in favour of FF/UMEC/VI was blunted in patients who continue to smoke.

Impact of 24-week CID status on 52-week outcomes in the EXT population

Across both treatment arms, the CID- subgroup (i.e. patients who had not experienced a CID event by 24 weeks) demonstrated sustained clinically important improvements from baseline in trough FEV1, SGRQ total score, CAT and E-RS:COPD at 52 weeks, in contrast to the CID+ subgroup. The incidence of moderate/severe exacerbations was similarly low in both subgroups in the 24-52-week follow-up assessment (CID-: $\mathrm{n}=19$ (13\%); CID+: $\mathrm{n}=38$ (15\%)) (table 3).

\section{Discussion}

As lung function, health status and exacerbations all contribute to the long-term prognosis in patients with COPD, management guidelines recommend that these parameters are considered when assessing disease progression and severity [1]. Previous studies have retrospectively evaluated the concept of CID to provide clinically relevant thresholds and quantify what proportion of patients with COPD achieve shorter term disease stability on standard-of-care mono- and dual therapy [8-10]. In this first prospective CID analysis in the FULFIL study, we have examined this concept with triple therapy versus ICS/LABA dual therapy in patients with symptomatic advanced COPD. Treatment with FF/UMEC/VI significantly reduced the risk of a first composite CID event by $47-52 \%$ and prolonged the median time to a first CID event by at least five-fold, compared with BUD/FOR. The increase in shorter term stability with FF/UMEC/VI versus BUD/ FOR provides additional insight on the overall treatment benefit of optimising lung function with a once daily, single-inhaler triple therapy compared with a twice daily ICS/LABA, in addition to the mean improvements in lung function and health status that have been previously reported [7].

The use of a composite CID end-point in the FULFIL trial to assess disease stability in patients with COPD is in line with the 2017 GOLD strategy. However, a potential limitation of this composite end-point approach is the lack of transparency in defining which individual CID event type was prevented. Importantly, in our study, the risk of each individual CID component event type was also reduced with FF/UMEC/VI versus BUD/FOR in the ITT population when using both CID definitions (SGRQ- and CAT-containing). The largest risk reduction in favour of FF/UMEC/VI for a single CID component event type was observed for $\mathrm{FEV}_{1}$, followed by exacerbations and, to a lesser degree, health status. As health status was monitored less frequently than FEV1, we also conducted a sensitivity analysis that censored deteriorations in FEV1 at visits where health status was not assessed. This sensitivity analysis showed 
a)

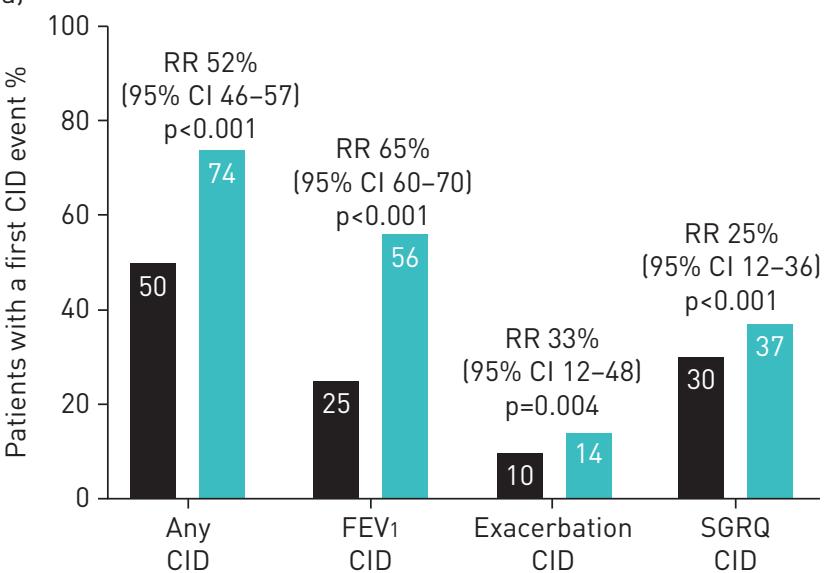

c)

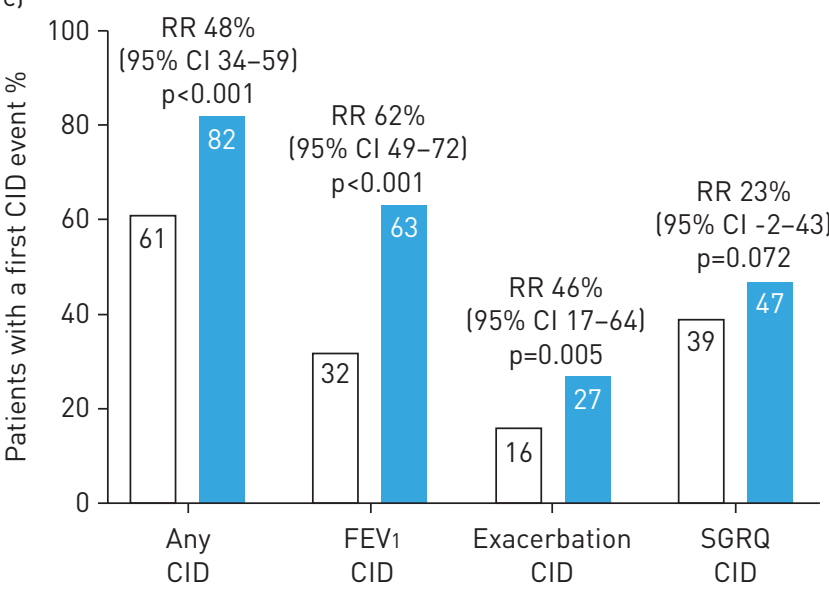

b)

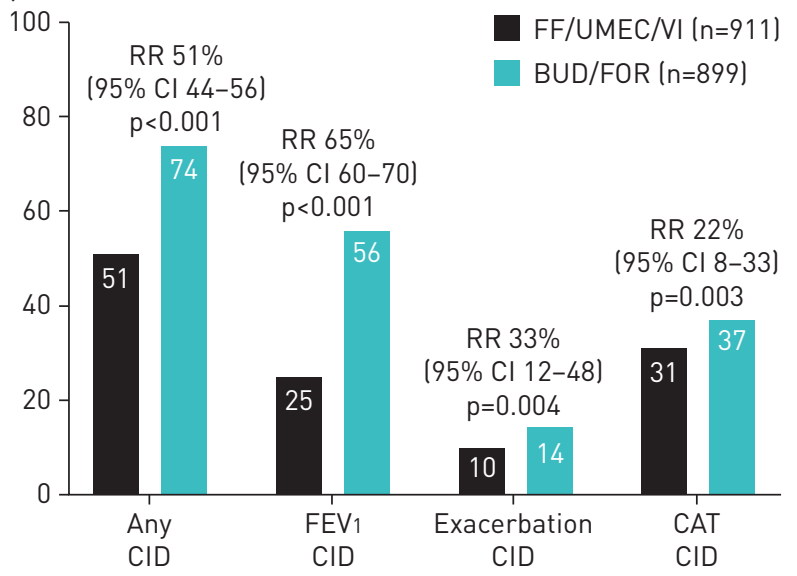

d)

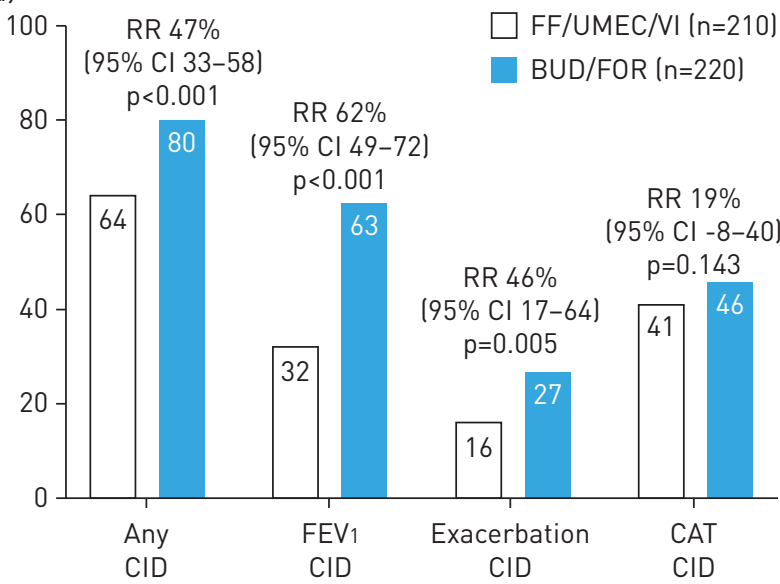

FIGURE 2 Occurrence of a clinically important deterioration (CID) by individual CID component event type in a, b) the intent-to-treat population (24-week study duration, $\mathrm{n}=1810$ ) and $\mathrm{c}$, d) the extension population (52-week study duration, $\mathrm{n}=430$ ). CID assessment using a, c) the St George's Respiratory Questionnaire (SGRQ)-containing definitions and b, d) chronic obstructive pulmonary disease assessment test (CAT)-containing definitions. FEV1: forced expiratory volume in $1 \mathrm{~s}$; FF: fluticasone furoate; UMEC: umeclidinium; VI: vilanterol; BUD: budesonide; FOR: formoterol; RR: relative risk (based on a time-to-first-event analysis using a Cox proportional hazards model).

similar findings to the ITT CID analyses with a significant reduction in the risk of a first composite CID event of $41-47 \%$ with FF/UMEC/VI compared with BUD/FOR.

The observed benefits with FF/UMEC/VI in the ITT 24-week assessment are similar to the results of a previous 3-month post hoc analysis of UMEC versus placebo when added to concurrent ICS/LABA therapy, which showed comparable significant benefits on lung function and exacerbation risk, and a smaller impact on health status, particularly in patients with a prior exacerbation history [14]. Taken together, these findings demonstrate the disease stabilising effect of aiming for maximal bronchodilation with single-inhaler FF/UMEC/VI or ICS/LABA plus UMEC using multiple inhalers, and highlight that these benefits occur early in patients with COPD.

Sensitivity analyses of the time to a first composite CID event in different patient subgroups confirmed the findings in the overall ITT population and were consistent across most of the subgroups (segmented by baseline severity of airway obstruction, health status impact, exacerbation history, sex and prior ICS and triple therapy at screening). We did, however, identify a significant interaction by smoking status, demonstrating a greater efficacy benefit in favour of FF/UMEC/VI for former smokers compared with current smokers. We propose that, in a non-smoking population, the efficacy of all components in the triple therapy, particularly the ICS, are likely to be optimal, thereby allowing for greater differentiation of the once daily combination versus twice daily BUD/FOR. Nevertheless, the finding that FF/UMEC/VI significantly reduced the risk of a first composite CID event in all subgroups tested demonstrates that no single subgroup drove the overall study findings, including those patients who stepped down from triple therapy at randomisation. Indeed, patients with moderate airflow limitation (GOLD grade 2), moderate 
a)

GOLD grade
$1 / 2$
$3 / 4$
Prior exacerbation history
$\leqslant 1$ exacerbation prior year
$\geqslant 2$ exacerbations prior year
Sex
Male
Female
Baseline CAT scores
$<20$
$\geqslant 20$
Triple therapy use at screening
Yes
No
ICS use at screening
Yes
No
Smoking status
Current
Former

b)

GOLD grade
$1 / 2$
$3 / 4$
Prior exacerbation history
$\leqslant 1$ exacerbation prior year
$\geqslant 2$ exacerbations prior year
Sex
Male
Female
Baseline CAT scores
$<20$
$\geqslant 20$
Triple therapy use at screening
Yes
No
ICS use at screening
Yes
No
Smoking status
Current
Former

)

Former

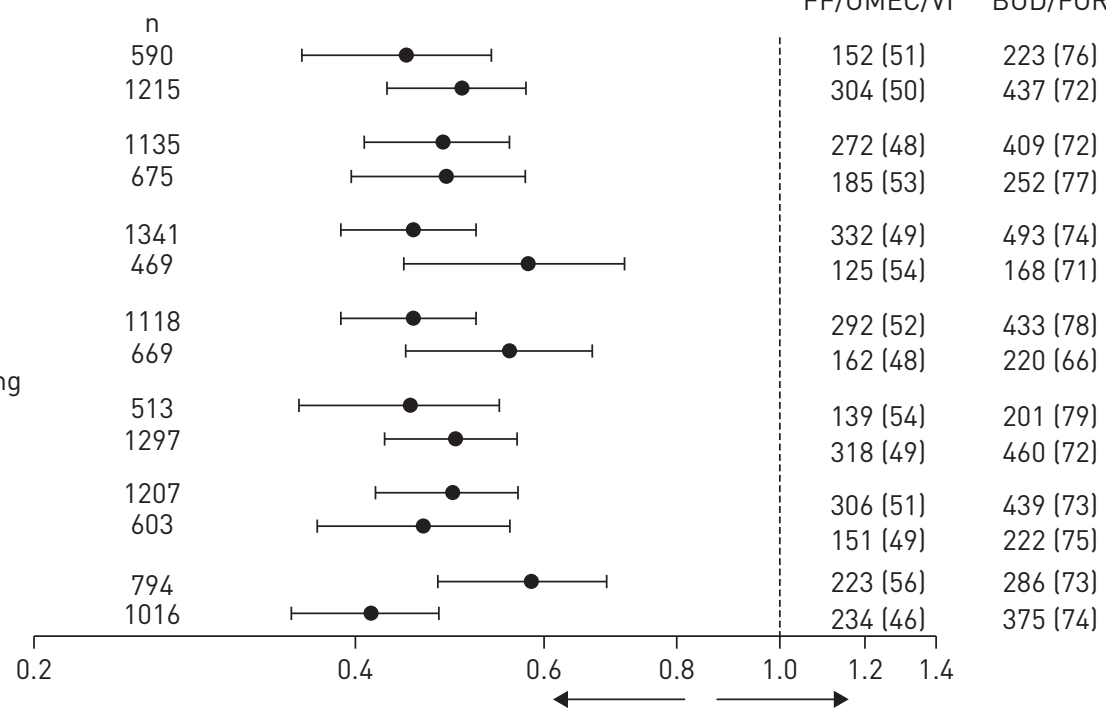

Risk increase with BUD/FOR Risk increase with FF/UMEC/VI Hazard ratio $(95 \% \mathrm{Cl})$

$$
\frac{\text { Patients with event } \mathrm{n}(\%)}{\text { FF/UMEC/VI } \quad \text { BUD/FOR }} \quad \begin{gathered}
\text { Hazard ratio } \\
(95 \% \mathrm{Cl})
\end{gathered}
$$

$0.44(0.36-0.54)^{* * *}$ $0.50(0.43-0.58)^{* * *}$

$0.48(0.41-0.56)^{* * *}$ $0.48(0.40-0.58)^{* * *}$

$0.45(0.39-0.52)^{* * *}$ $0.57(0.45-0.72) * * *$

$0.45(0.39-0.52)^{* * *}$ $0.55(0.45-0.67)^{* * *}$

$0.44(0.36-0.55)^{* * *}$

$0.49(0.43-0.57)^{* * *}$

$0.49(0.42-0.57)^{* * *}$

$0.46(0.37-0.56)^{* * *}$

$0.58(0.48-0.69)^{* * *}$

$0.41(0.35-0.48)^{* * *}$

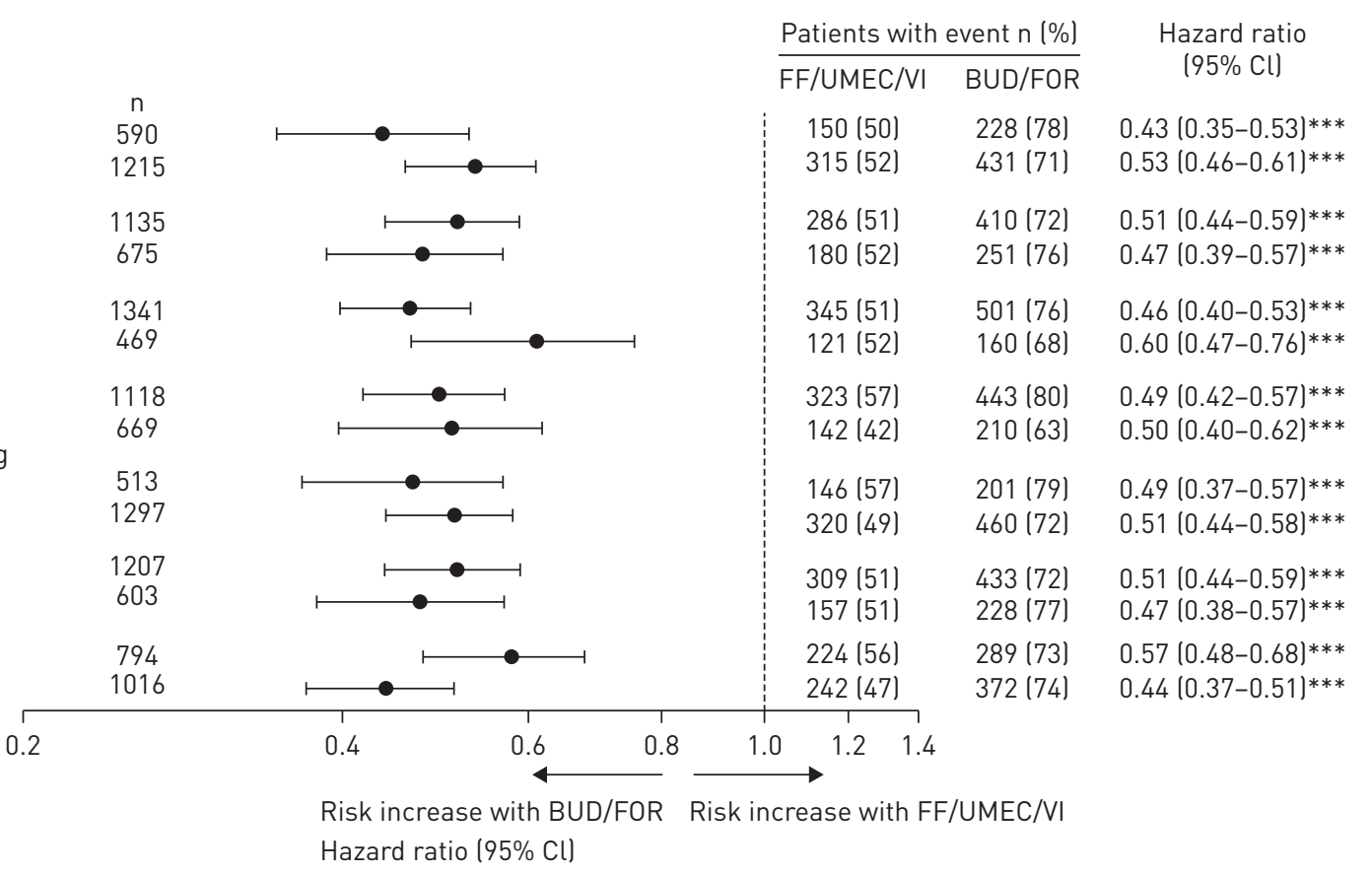

Patients with event $\mathrm{n}(\%)$

zard ratio FEMTECN $150(50) \quad 228(78)$ $431(71)$

$0.43(0.35-0.53)^{* * *}$ $0.53(0.46-0.61)^{* * *}$

$286(51) \quad 410(72) \quad 0.51(0.44-0.59)^{* * *}$ $345(51) \quad 501(76) \quad 0.46(0.40-0.53)^{* * *}$ $121(52) \quad 160(68) \quad 0.60(0.47-0.76)^{* * *}$ $323(57) \quad 443(80) \quad 0.49(0.42-0.57)^{* * *}$ $142(42) \quad 210(63) \quad 0.50(0.40-0.62)^{* * *}$

$146(57) \quad 201(79) \quad 0.49(0.37-0.57)^{* * *}$ $460(72) \quad 0.51(0.44-0.58)^{* * *}$

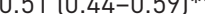
$228(77) \quad 0.47(0.38-0.57)^{* * *}$ $89(73) \quad 0.57(0.48-0.68) * * *$ $0.44(0.37-0.51)^{* * *}$

Risk increase with BUD/FOR Risk increase with FF/UMEC/VI Hazard ratio $(95 \% \mathrm{Cl})$

FIGURE 3 Forest plot of hazard ratios for time to first composite clinically important deterioration (CID) event segmented by baseline disease severity, exacerbation history, sex, health status and prior treatment at screening using a) the St George's Respiratory Questionnaire-containing and b) the chronic obstructive pulmonary disease assessment test (CAT)-containing CID definitions (intent-to-treat population). Hazard ratios and 95\% $\mathrm{Cls}$ are from a Cox proportional hazards model with covariates of treatment group, smoking status at screening and geographical region, plus the specified factor and specified factor by treatment group interaction. GOLD: Global Initiative for Chronic Obstructive Lung Disease; ICS: inhaled corticosteroid; FF: fluticasone furoate; UMEC: umeclidinium; VI: vilanterol; BUD: budesonide; FOR: formoterol. ${ }^{* * *}$ : $p<0.001$ (post hoc sensitivity analysis).

health status impact $(\mathrm{CAT}<20)$ and lower exacerbation risk $(\leqslant 1$ event per year) all obtained similar or greater levels of risk reduction in time to a first composite CID event with FF/UMEC/VI versus BUD/FOR to the overall ITT population. This implies that optimisation of lung function may be an important first treatment goal in all symptomatic patients with COPD (i.e. GOLD groups B and D).

To further assess the prognostic benefit of shorter term disease stability in the FULFIL trial, we segmented patients who had or had not experienced a CID by 24 weeks in the EXT population. Stable patients (i.e. those who had not experienced a CID at 24 weeks) had similar baseline characteristics to those with a CID 


\begin{tabular}{|c|c|c|}
\hline Outcome measure & CID- subgroup & CID+ subgroup \\
\hline Subjects $\mathbf{n}$ & 152 & 260 \\
\hline \multicolumn{3}{|l|}{ Trough FEV $1 \mathrm{~mL}$} \\
\hline Baseline & 129 & 223 \\
\hline Mean \pm SD & $1421 \pm 536$ & $1356 \pm 575$ \\
\hline Change from baseline at week 52 & 129 & 223 \\
\hline Mean $\pm S D$ & $169 \pm 242$ & $-31 \pm 245$ \\
\hline \multicolumn{3}{|l|}{ SGRQ total score $\#$} \\
\hline Baseline $\mathrm{n}$ & 152 & 259 \\
\hline Mean $\pm S D$ & $55.4 \pm 15.9$ & $49.8 \pm 15.2$ \\
\hline Change from baseline at week $52 \mathrm{n}$ & 130 & 223 \\
\hline Mean $\pm S D$ & $-10.1 \pm 14.1$ & $1.2 \pm 14.1$ \\
\hline \multicolumn{3}{|l|}{ CAT $^{\#}$} \\
\hline Baseline & 151 & 258 \\
\hline Mean \pm SD & $19.1 \pm 6.6$ & $17.2 \pm 5.5$ \\
\hline Change from baseline at week 52 & 129 & 222 \\
\hline Mean $\pm S D$ & $-3.6 \pm 6.4$ & $-0.2 \pm 5.8$ \\
\hline \multicolumn{3}{|l|}{ E-RS:COPD ${ }^{\#}$} \\
\hline Baseline $\mathrm{n}$ & 151 & 258 \\
\hline Mean $\pm S D$ & $14.20 \pm 5.55$ & $12.88 \pm 5.46$ \\
\hline Change from baseline at week 52 & 130 & 223 \\
\hline Mean $\pm S D$ & $-3.14 \pm 5.65$ & $-0.83 \pm 5.17$ \\
\hline Incidence of moderate/severe COPD exacerbations ${ }^{\pi}$ & $19(13)$ & $38(15)$ \\
\hline \multicolumn{3}{|c|}{$\begin{array}{l}\text { Data are presented as } n \text { or } n(\%) \text {, unless otherwise stated. CID: clinically important deterioration; EXT: } \\
\text { extension; FEV1: forced expiratory volume in } 1 \mathrm{~s} \text {; SGRQ: St George's Respiratory Questionnaire; COPD: } \\
\text { chronic obstructive pulmonary disease; CAT: COPD assessment test; E-RS:COPD: Evaluating Respiratory } \\
\text { Symptoms in COPD. \#: a decrease in score represents an improvement in symptoms. }{ }^{\#} \text { : incidence } \\
\text { assessed post-CID assessment during weeks } 25 \text { to } 52 \text {. The CID- subgroup included patients who had not } \\
\text { experienced a CID at } 24 \text { weeks; the CID+ subgroup included patients who had experienced a CID at } \\
24 \text { weeks. }\end{array}$} \\
\hline
\end{tabular}

by 24 weeks; however, the CID- subgroup had sustained clinically important improvements in lung function, health status and overall symptoms at 52 weeks, in contrast to the CID+ subgroup. This indicates that early monitoring of disease stability may be a useful predictor of future prognosis with sustained clinically important benefits only seen in patients who do not experience an early CID event.

This structured monitoring of disease stability may also provide additional insight on future exacerbation risk. This was not supported by our shorter term descriptive analysis of exacerbation incidence in the CID- and CID+ subgroups in the small EXT population during weeks 24-52 of follow-up; however, three previous retrospective analyses showed an association between the shorter term occurrence of a CID event and an increased risk of future exacerbations and all-cause mortality in the next 6-42 months of follow-up [15-17]. In an integrated post hoc CID analysis of the TORCH study population, conducted across all four treatment arms, patients experiencing a composite CID event in the first 6 months experienced sustained deterioration in FEV1 and health status, with an increased risk of exacerbations and mortality over the next 30 months [15]. Similarly, a more recent analysis of TORCH showed that patients on the best treatment arm in the trial, i.e. ICS/LABA therapy, with a CID in the first 6 months also showed very similar increased risk of exacerbations requiring hospitalisations and all-cause mortality compared with patients without a CID event [16]. In these analyses, the greatest survival was seen in patients free of all CID types [17]. Post hoc analyses from the UPLIFT and ECLIPSE trials also confirmed the prognostic value of composite CID events at 6 and 12 months, respectively, in identifying patients at increased risk of mortality $[17,18]$. In UPLIFT, the predictive value of individual CID components differed according to the type of long-term outcome and the timing of the CID event $[18,19]$. When patients with CID events in UPLIFT were compared to those without a single event at both 6 and 12 months, CID events predicted a $21 \%$ and $31 \%$ increase in all-cause mortality, respectively. However, using a time-varying covariate to predict death with a CID event at any time-point was associated with a $69 \%$ significant increase in mortality [19]. SGRQ as a CID event was the strongest individual predictor of future mortality in TORCH [17] and, in ECLIPSE, patients with a 1-year deterioration in health status measured by SGRQ at the CID level were also the most likely to have increased risk of hospitalisations and mortality [20]. Surprisingly, an improvement in SGRQ had no bearing on future exacerbation risk or mortality [20]. With regard to 
deterioration in FEV1, lung function variability is known to be an important predictor of hospitalisation risk and mortality at the population level, although the importance of considering symptoms and exacerbation history alongside FEV1 is recognised when considering individualised patient care $[1,21]$. Together, this evidence suggests that the composite CID end-point may be an effective tool for predicting longer term outcomes in patients with COPD.

The generalisability of the CID concept in this first prospective analysis was potentially enhanced by replacing the SGRQ component of the composite CID end-point with deterioration in CAT score, which provided similar results. This is an important finding as the CAT instrument is easier to administer and interpret in the context of clinical practise than the SGRQ, and may facilitate the assessment of health status deterioration when using CID outside of a research setting. Indeed, our analysis suggests that, in the future, the CAT score could replace the SGRQ total score as the measure of health status in the CID end-point.

A potential limitation of this analysis was that the comparator group in the FULFIL trial received ICS/ LABA therapy and the study lacked an additional LAMA/LABA combination arm. Therefore, we were unable to address the question of how the ICS component contributed to the observed beneficial effect of FF/UMEC/VI on CID. However, this question will be addressed in the forthcoming IMPACT trial, which compared FF/UMEC/VI with both FF/VI and UMEC/VI [22]. Another potential study limitation was that health status assessments had unequal weighting to the contribution made by trough FEV1 in the composite CID. To mitigate for this, we performed a sensitivity analysis that censored additional FEV 1 data collected at visits where health status was not assessed, which showed similar findings to the ITT analyses. Nevertheless, more frequent health status assessments would be a preferred solution in future trials. Finally, the small EXT population used to assess the prognostic link between shorter term stability and longer term outcomes experienced too few exacerbations to provide firm conclusions regarding the occurrence of shorter term CID and future exacerbation risk. Therefore, we remain reliant on previous retrospective analyses to substantiate this link [15-17].

\section{Conclusions}

This first prospective CID analysis in the FULFIL study showed that once daily, single-inhaler triple therapy with FF/UMEC/VI significantly reduced the risk of CID in patients with COPD, compared with twice daily BUD/FOR. Our results support the concept of using CID as a prospective end-point in COPD clinical studies and highlight the importance of maximising lung function at an early stage of treatment in patients with advanced disease. These findings also support management guidelines on the need for regular monitoring of potential disease progression in COPD across multiple end-points, particularly the wider adoption of the CAT score as a measure of health status in addition to assessing lung function and exacerbations.

Acknowledgements: We would like to thank the patients and their families for participating in the FULFIL study, Helen Barnacle (GSK Clinical Investigational Leader), Eva Gomez (GSK Operations Lead), Erik Steinberg (GSK Data Quality Leader), Niki Day and Rajat Mohindra (GSK Global Clinical Pharmacovigilance), and the FULFIL study team. Medical writing support in the form of development of the draft outline and manuscript drafts in consultation with the authors, editorial suggestions to draft versions of this paper, assembling tables and figures, collating author comments, copyediting, referencing and graphic services was provided by Thomas Burton (Gardiner-Caldwell Communications, Macclesfield, UK) and was funded by GSK.

Conflict of interest: I. Naya is an employee of GSK and holds stocks/shares in GSK. C. Compton is an employee of GSK and holds stocks/shares in GSK. A.S. Ismaila is an employee of GSK and holds stocks/shares in GSK and is an unpaid faculty member at McMaster University, Canada. R. Birk is an employee of GSK and holds stocks/shares in GSK. N. Brealey is an employee of GSK and holds stocks/shares in GSK. M. Tabberer is an employee of GSK and holds stocks/ shares in GSK. C-Q. Zhu is an employee of GSK and holds stocks/shares in GSK. D.A. Lipson is an employee of GSK and holds stocks/shares in GSK. G. Criner reports grants from Boehringer Ingelheim, Novartis, AstraZeneca, Respironics, MedImmune, Actelion, Forest, Pearl, Ikaria, Aeris, PneumRx and Pulmonx, as well as equity interest from HGE Health Care Solutions, Inc, and consultation fees from Amirall, Boehringer Ingelheim and Holaira, outside the submitted work.

This study was funded by GSK (ClinicalTrials.gov NCT02345161; GSK study CTT116853). ELLIPTA is owned by or licensed to the GSK group of companies. Turbuhaler is a registered trade mark of AstraZeneca. Information on GSK's data sharing commitments and requesting access to anonymised individual participant data and associated documents can be found at www.clinicalstudydatarequest.com.

\section{References}

1 Global Initiative for Chronic Obstructive Lung Disease (GOLD). GOLD Global Strategy for the Diagnosis, Management and Prevention of COPD. 2017. http://goldcopd.org/gold-2017-global-strategy-diagnosismanagement-prevention-copd/ Date last accessed: October 10, 2017.

2 Aaron SD, Vandemheen KL, Fergusson D, et al. Tiotropium in combination with placebo, salmeterol, or fluticasone-salmeterol for treatment of chronic obstructive pulmonary disease: a randomized trial. Ann Intern Med 2007; 146: 545-555.

3 Welte T, Miravitlles M, Hernandez P, et al. Efficacy and tolerability of budesonide/formoterol added to tiotropium in patients with chronic obstructive pulmonary disease. Am J Respir Crit Care Med 2009; 180: 741-750. 
4 Siler TM, Kerwin E, Tombs L, et al. Triple therapy of umeclidinium+inhaled corticosteroids/long-acting $\beta_{2}$-agonists for patients with COPD: pooled results of randomized placebo-controlled trials. Pulm Ther 2016; 2 : 43-58.

5 Singh D, Papi A, Corradi M, et al. Single inhaler triple therapy versus inhaled corticosteroid plus long-acting $\beta_{2}$-agonist therapy for chronic obstructive pulmonary disease (TRILOGY): a double-blind, parallel group, randomised controlled trial. Lancet 2016; 388: 963-973.

6 Vestbo J, Papi A, Corradi M, et al. Single inhaler extrafine triple therapy versus long-acting muscarinic antagonist therapy for chronic obstructive pulmonary disease (TRINITY): a double-blind, parallel group, randomised controlled trial. Lancet 2017; 389: 1919-1929.

7 Lipson DA, Barnacle H, Birk R, et al. FULFIL trial: once-daily triple therapy for patients with chronic obstructive pulmonary disease. Am J Respir Crit Care Med 2017; 196: 438-446.

8 Singh D, Maleki-Yazdi MR, Tombs L, et al. Prevention of clinically important deteriorations in COPD with umeclidinium/vilanterol. Int J Chron Obstruct Pulmon Dis 2016; 11: 1413-1424.

9 Maleki-Yazdi MR, Singh D, Anzueto A, et al. Assessing short-term deterioration in maintenance-naïve patients with COPD receiving umeclidinium/vilanterol and tiotropium: a pooled analysis of three randomized trials. $A d v$ Ther 2017; 33: 2188-2199.

10 Anzueto AR, Vogelmeier CF, Kostikas K, et al. The effect of indacaterol/glycopyrronium versus tiotropium or salmeterol/fluticasone on the prevention of clinically important deterioration in COPD. Int J Chron Obstruct Pulmon Dis 2017; 12: 1325-1337.

11 Jones PW, Harding G, Berry P, et al. Development and first validation of the COPD Assessment Test. Eur Respir J 2009; 34: 648-654.

12 Jones PW, Harding G, Wiklund I, et al. Tests of the responsiveness of the COPD assessment test following acute exacerbation and pulmonary rehabilitation. Chest 2012; 142: 134-140.

13 Miller MR, Hankinson J, Brusasco V, et al. Standardisation of spirometry. Eur Respir J 2005; 26: 319-338.

14 Naya IP, Lipson DA, Compton C. Prevention of early worsening of COPD with umeclidinium open triple therapy compared with inhaled corticosteroid/long-acting $\beta 2$-agonist alone: a pooled post hoc analysis. Am J Respir Crit Care Med 2017; 195: A3608.

15 Naya IP, Tombs L, Jones P. Short-term clinically important deterioration predicts long-term clinical outcome in COPD patients: a post-hoc analysis of the TORCH trial. Thorax 2015; 70: Suppl. 3, A34.

16 Naya IP, Driessen MT, Paly V, et al. Long-term consequences of clinically important deterioration in patients with chronic obstructive pulmonary disease treated with twice-daily inhaled corticosteroid/long-acting $\beta 2$-agonist therapy: results from the TORCH study. Am J Respir Crit Care Med 2018; 197: A3042.

17 Naya IP, Tombs L, Mullerova $\mathrm{H}$, et al. Long-term outcome following first clinically important deterioration in COPD. Eur Respir J 2016; 48: PA304.

18 Rabe KF, Halpin D, Martinez F, et al. Predicting long-term outcomes and future deterioration in COPD with a composite endpoint: post hoc analysis of the UPLIFT study. Am J Respir Crit Care Med 2017; 195: A2717.

19 Rabe KF, Halpin D, Martinez F, et al. Relative timing of clinically important deterioration and related long-term outcomes in COPD: a post hoc analysis of the UPLIFT study. Pneumologie 2018; 72: S86-S87.

20 Wilke S, Jones PW, Müllerova $\mathrm{H}$, et al. One-year change in health status and subsequent outcomes in COPD. Thorax 2015; 70: 420-425.

21 So JY, Lastra AC, Zhao H, et al. Daily peak expiratory flow rate and disease instability in chronic obstructive pulmonary disease. Chronic Obstr Pulm Dis 2015; 3: 398-405.

22 Pascoe SJ, Lipson DA, Locantore N, et al. A phase III randomised controlled trial of single-dose triple therapy in COPD: the IMPACT protocol. Eur Respir J 2016; 48: 320-330. 